\title{
Thiomethyl Derivatives of Two Oligo(p-phenylene vinylenes) as New Aggregation-Induced Piezofluorochromic Emitters
}

\author{
Xueqing Hou ${ }^{1}$, Jing Ling ${ }^{1}$, Navamoney Arulsamy ${ }^{2}$, Jianqiang Huo ${ }^{1 *}$ \\ ${ }^{1}$ Key Laboratory of Eco-Environment Related Polymer Materials of Ministry of Education, Key Laboratory of Polymer Materials of \\ Gansu Province, College of Chemistry and Chemical Engineering, Northwest Normal University, Lanzhou, China; ${ }^{2}$ Department of \\ Chemistry, University of Wyoming, Laramie, USA. \\ Email: ${ }^{*}$ huojianqiang@nwnu.edu.cn
}

Received April $2^{\text {nd }}, 2013$; revised May $3^{\text {rd }}, 2013$; accepted May $12^{\text {th }}, 2013$

Copyright (C) 2013 Xueqing Hou et al. This is an open access article distributed under the Creative Commons Attribution License, which permits unrestricted use, distribution, and reproduction in any medium, provided the original work is properly cited.

\begin{abstract}
Two piezochromic fluorescent compounds are prepared by introducing thiomethyl substituents to the peripheralpositions of two related cyano oligo(p-phenylene vinylenes) (CN-OPV). The new derivatives, namely, 1,4-bis[2-cyano-2(4-thiomethylphenyl)ethenyl]benzene (TOPV1) and 1,4-bis(1-cyano-2-(4-thiomethylphenyl)ethenyl) benzene (TOPV2), are characterized by NMR, powder X-ray diffraction data (XRD) and differential scanning calorimetric (DSC) data. UV-Vis and fluorescence spectra are also measured. TOPV1 with the cyano groups farther away from the central aromatic ring is photoluminescent, and on application of pressure exhibits a more obvious color change and higher Stokes shift than those measured for TOPV2. We also observe piezochromic aggregation-induced emission (PAIE) for TOPV2, but TOPV1 does not exhibit any PAIE.
\end{abstract}

Keywords: Aggregation Induced Emission (AIE); Piezochromic Fluorophore; Excimer Fluorescence

\section{Introduction}

The development of new fluorescent organic materials is of great interest for both fundamental research and practical applications. Certain fluorescent materials that also exhibit color changes due to pressure-dependent molecular packing of their constituent molecules are known as piezofluorochromic emitters. Recently, various examples of mechano-responsive polymeric materials and lightinduced fluorescence switching compounds and have been reported [1-4]. These materials exhibit luminescent color change as a result of molecular aggregation or stacking. Among them, compounds showing piezochromic properties are drawing special attentions in view of developing functional sensors [5-9], memory chips [10,11], optical switches [12,13], security inks [14], and fluorescent probes [15].

Cyano derivatives of oligo(p-phenylene vinylene) (OPV) are well known for their luminescent and piezochromic properties [2,16-21]. For example, Chung et al., have reported the presence of reversible fluorescence switch-

*Corresponding author. ing in cyano-stilbene derivatives. These molecules pack head-to-tail in their crystals with their stilbenic double bonds being aligned parallel. The center-to-center distances between the double bonds of two neighboring molecules are shorter at ca $3.8 \AA$, and the $\pi$-dimer formation is attributed to the intermolecular C-F $\cdots \mathrm{H}$ and C-F $\cdots \pi$ interactions caused by the highly electronegative $\mathrm{CF}_{3}$ substituents. Significantly, the $\pi$-dimeric arrangement in the crystals is suggested to undergo lateral displacement either by shear-strain or UV irradiation causing the rare fluorescence switching observed for the crystals [2].

The cyano groups present in the OPV's and related compounds [2,9-12] assist in the formation of a Donor- $\pi$-Acceptor (D- $\pi$-A) conjugate system. We expected that the position of the cyano substituent may have an effect on the photoluminescent properties of the OPV's as the cyano substitution at the 1-position of the vinyl groups versus the 2-position will significantly change the length of the $\pi$-bridge. Surprisingly, no studies on isomeric CN-OPV's with varying $\pi$-bridge lengths are known in the literature. Therefore, we have undertaken 
the synthesis and studies of a CN-OPV system, namely, bis(1- or 2-cyano)-2-(4-thiomethylphenyl)benzene. We also expected enhanced aggregation-induced emission (AIE) due to the electron-donating nature of the thiomethyl groups should increase AIE effect dramatically. Herein, we report first investigation of the effect of cyano substituents at either the 1- or 2-positions of the vinyl group on the overall photoluminescent property of the thiomethylphenyl cyano oligo(phenylene vinylenes), TO-PV1 and TOPV2 (Figure 1).

\section{Methodology}

Terephthalaldehyde, (4-methylthiophenyl)acetonitrile, 4(thiomethyl)benzalde-hyde purchased from J\&K were used as received. All other reagents and solvents were purchased as analytical grade from Beijing Special Chemicals Co. (China) and used without further purification. Tetrahydrofuran (THF) was distilled from sodium/benzophenone. Ultrapure water was used in the spectroscopic measurements.

The ${ }^{1} \mathrm{H}$ and ${ }^{13} \mathrm{C}$ NMR spectra were measured on a JEM-ECP400 NMR spectrometer using $\mathrm{CDCl}_{3}$ as solvent containing tetramethylsilane (TMS) as the internal standard. Fluorescence spectra were measured on a Shimadzu RF-5301pc spectrometer with a slit width of $1.5 \mathrm{~nm}$ for both excitation and emission. Differential scanning calorimeter (DSC) curves were obtained with a NETZSCH thermal analyzer (DSC 204 F1) at heating and cooling rates of $10^{\circ} \mathrm{C}$ per min under $\mathrm{N}_{2}$ atmosphere. Wide-angle $\mathrm{X}$-ray diffraction (WAXD) measurements were performed on a Rigaku X-ray diffractometer (D/max-2400) with a $\mathrm{Cu} \operatorname{KR}(\lambda=0.15406 \mathrm{~nm}) \mathrm{X}$-ray tube at $40 \mathrm{kV}$ and 30 $\mathrm{mA}$ at a scan rate of $4^{\circ}(2 \theta)$ per min. The samples were ground using a mortar and pestle and pressed into pellets using a pellet press at $1500 \mathrm{psi}$ for $5 \mathrm{~min}$. Annealing experiments were done on a hot stage with automatic temperature control.
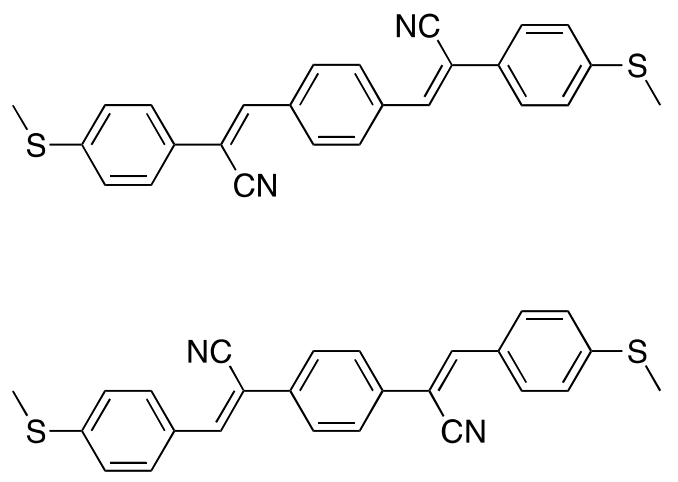

Figure 1. Molecular structures of TOPV1 (top) and TOPV2 (bottom).

\section{Syntheses}

1,4-bis[2-cyano-2-(4-thiomethylphenyl)ethenyl]-benze ne (TOPV1). A solution of terephthalaldehyde (268.0 $\mathrm{mg}, \quad 2.0 \mathrm{mmol}$ ) and (4-methylthiophenyl)acetonitrile $(652.0 \mathrm{mg}, 4.0 \mathrm{mmol})$ in t-BuOH $(25 \mathrm{~mL})$ was heated to $60^{\circ} \mathrm{C}$. To the solution was added t-BuOK $(504.0 \mathrm{mg}, 4.5$ $\mathrm{mmol}$ ) in one portion with stirring. A yellow precipitate started to form immediately. The mixture was stirred for $30 \mathrm{~min}$ at $60^{\circ} \mathrm{C}$, cooled to r.t., and poured into acidified methanol $(50 \mathrm{~mL}$ containing 1 drop of concentrated acetic acid). The precipitate was filtered, washed with excess methanol, and dried in vacuo at $50^{\circ} \mathrm{C}$. Yield: 720.8 $\mathrm{mg}(85 \%)$; yellow powder. ${ }^{1} \mathrm{H}$ NMR (400 $\mathrm{MHz}, \mathrm{CDCl}_{3}$ ) $\delta$ [ppm]: 7.64 (s, 2H, vinyl-H), $7.62(\mathrm{~d}, 4 \mathrm{H}, \mathrm{Ar}-\mathrm{H}), 7.51$ (s, 4H, Ar-H), 7.32 (d, 4H, Ar-H), 2.47 (s, $6 \mathrm{H})$.

1,4-bis(1-cyano-2-(4-thiomethylphenyl)ethenyl)benz ene (TOPV2). (4-Cyano-methylphenyl)acetonitrile (312 $\mathrm{mg}, 2.0 \mathrm{mmol}$ ) and 4-(thiomethyl) benzaldehyde (608 $\mathrm{mg}, 4.0 \mathrm{mmol})$ were dissolved in $\mathrm{t}-\mathrm{BuOH}(25 \mathrm{~mL})$ and the mixture was heated to $60^{\circ} \mathrm{C}$. $\mathrm{t}-\mathrm{BuOK}(504.0 \mathrm{mg}, 4.5$ $\mathrm{mmol}$ ) was added in one portion. The yellow precipitate formed was treated as above. Yield: $737.8 \mathrm{mg}(87 \%)$; yellow powder. ${ }^{1} \mathrm{H}$ NMR $(400 \mathrm{MHz}, \mathrm{CDCl} 3) \delta$ [ppm]: 7.54 (s, 2H, vinyl-H), $7.42(\mathrm{~d}, 4 \mathrm{H}, \mathrm{Ar}-\mathrm{H}), 7.32(\mathrm{~s}, 4 \mathrm{H}$, Ar-H), 7.18 (d, 4H, Ar-H), 2.47 (s, 6 H).

\section{Results and Discussion}

Positional isomers TOPV1 and TOPV2 are synthesized from the reaction of suitably substituted acetonitriles and aromatic aldehydes as shown in Figure 2. Both compounds are isolated as yellow powders and are soluble in solvents such as dichloromethane and chloroform. The compounds are readily characterized by their ${ }^{1} \mathrm{H}$ and ${ }^{13} \mathrm{C}$ NMR spectra. Recrystallization of the products from a variety of solvents formed microcrystals. DSC curves measured for the compounds reveal that they possess very high glass transition temperatures $(\mathrm{Tg})$. The melting point $(\mathrm{Tm})$ of TOPV1 is $276^{\circ} \mathrm{C}$. The Tm of TOPV2 is $257^{\circ} \mathrm{C}$.

We examined the effect of pressure on the two compounds. As shown in Figure 3, both dyes TOPV1 and TOPV2 exhibit reversible color change when under pressure or while grinding. The samples revert to their originnal color when heating is applied. The change of colors is more distinct for TOPV1 compared to that of TOPV2 (Figure 3). Further investigations indicate that dye TOPV2 exhibits aggregation-induced emission, whereas dye TOPV1 does not seem to exhibit AIE.

UV-Vis spectra measured for the compounds exhibit a single peak in dichloromethane solvent as shown in Figure 4 for TOPV1. The spectrum for TOPV2 measured in THF exhibits solvatochromic effect as the peak splits 

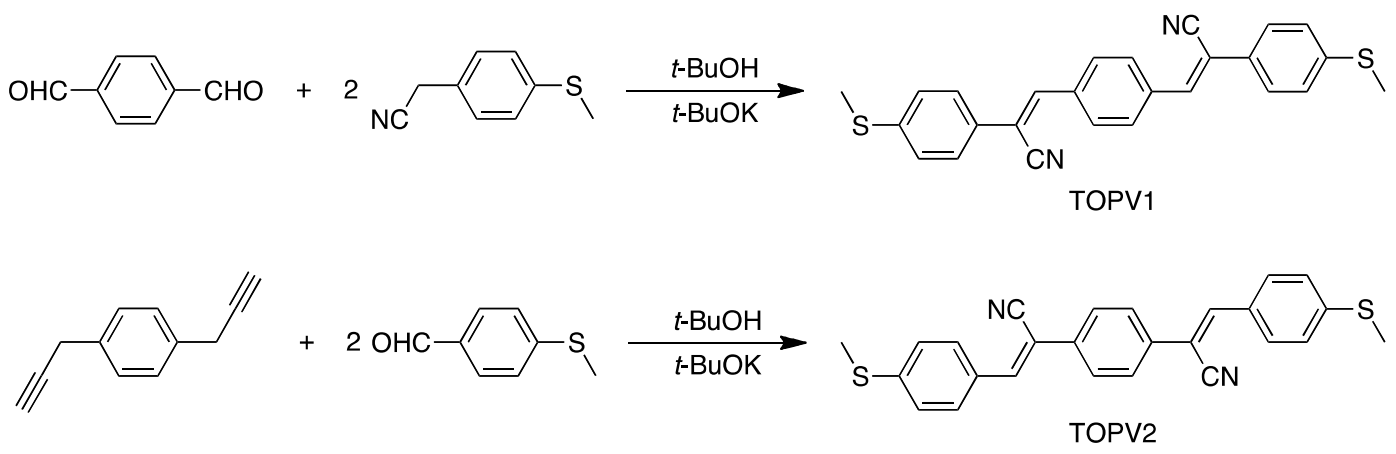

Figure 2. Synthetic routes for TOPV1 and TOPV2.

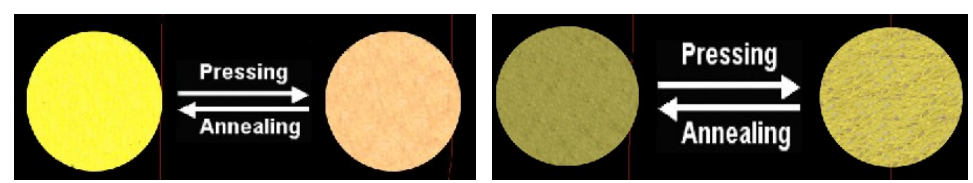

Figure 3. Image of TOPV1 and TOPV2.
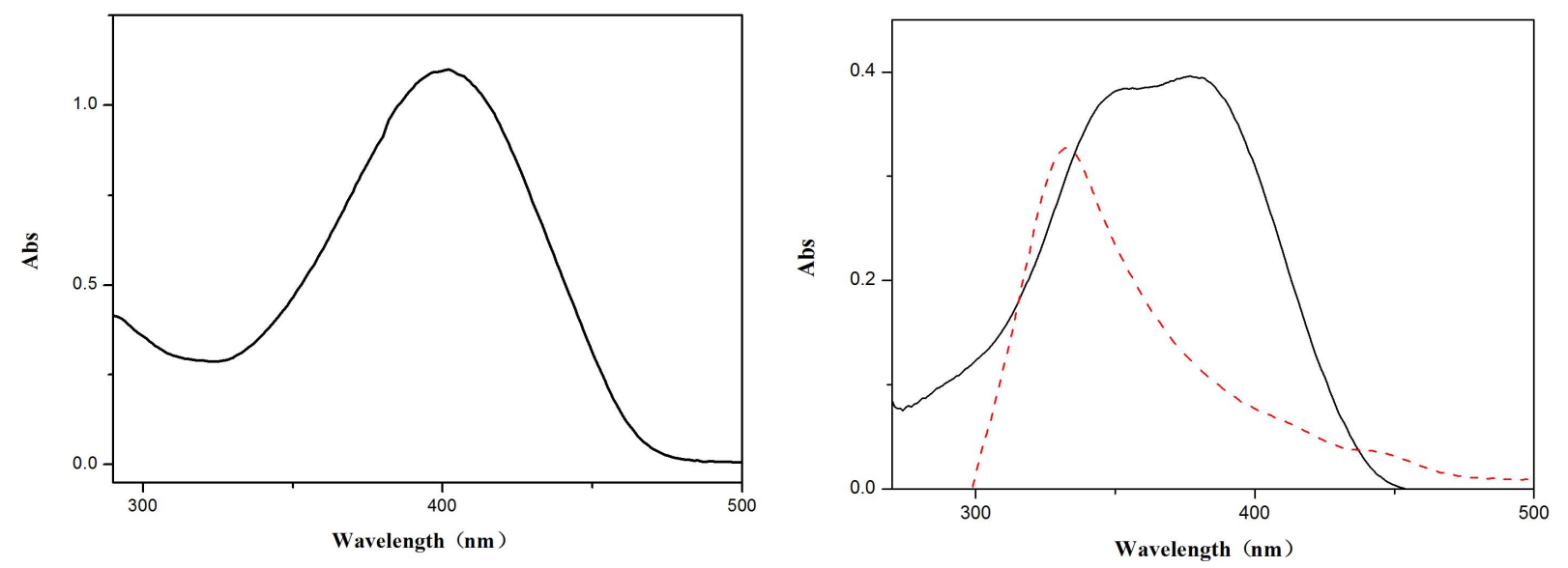

Figure 4. UV-Vis spectra for TOPV 1 in $\mathrm{CH}_{2} \mathrm{Cl}_{2}$ (left) and TOPV2 in THF solution (right, $\mathrm{c}=2 \times 10^{-5} \mathrm{~mol} \cdot \mathrm{L}^{-1}$, black solid line) and TOPV2 in 50:50 THF/water mixture (red broken line, $\mathrm{c}=2 \times 10^{-5} \mathrm{~mol} \cdot \mathrm{L}^{-1}$ ).

into two overlapping peaks in THF. The spectrum exhibits further change in a 50:50 THF:water solvent mixture. A single peak is observed at a considerably high energy indicating PAIE.

Figure 5 shows the photoluminescence (PL) spectra for the samples obtained under different conditions (pressing and annealing). The emission maxima exhibit significant red shift, as high as $53 \mathrm{~nm}$, after pressing. After treatment of the pressed samples through annealing, the color returned to that of the original state, and the process is reversible. The reversible color change feature makes TOPV1 a promising candidate for light- or heat- or pressure-sensing applications. The observed Stokes shift of $95 \mathrm{~nm}$ demonstrates a great photoluminescence property for TOPV1.

In order to further explore the solid-state structures of the two TOPV dyes, powder X-ray diffraction experi- ments were conducted. The measured diffraction patterns display sharp peaks indicative of crystalline order. The XRD plots for samples that were well ground exhibit a series of new peaks, indicating significant change in the molecular packing (Figure 6). Subsequently, the ground samples were annealed by heating to $180^{\circ} \mathrm{C}$ for $5 \mathrm{~min}$, and their XRD patterns measured. The XRD plots for the annealed samples are nearly identical to the plots for the original samples, indicating the restoration of their original packing in the crystals.

To determine whether the compound is AIE active, the fluorescent behaviors of diluted mixtures were studied in mixtures of $\mathrm{THF} /$ water in various proportions. These experiments reveal no AIE for TOPV1. However, the fluorescence data for TOPV2 suggest both piezofluorochromic and AIE effects. As TOPV2 is insoluble in water, increasing the water fraction in the mixed solvent 

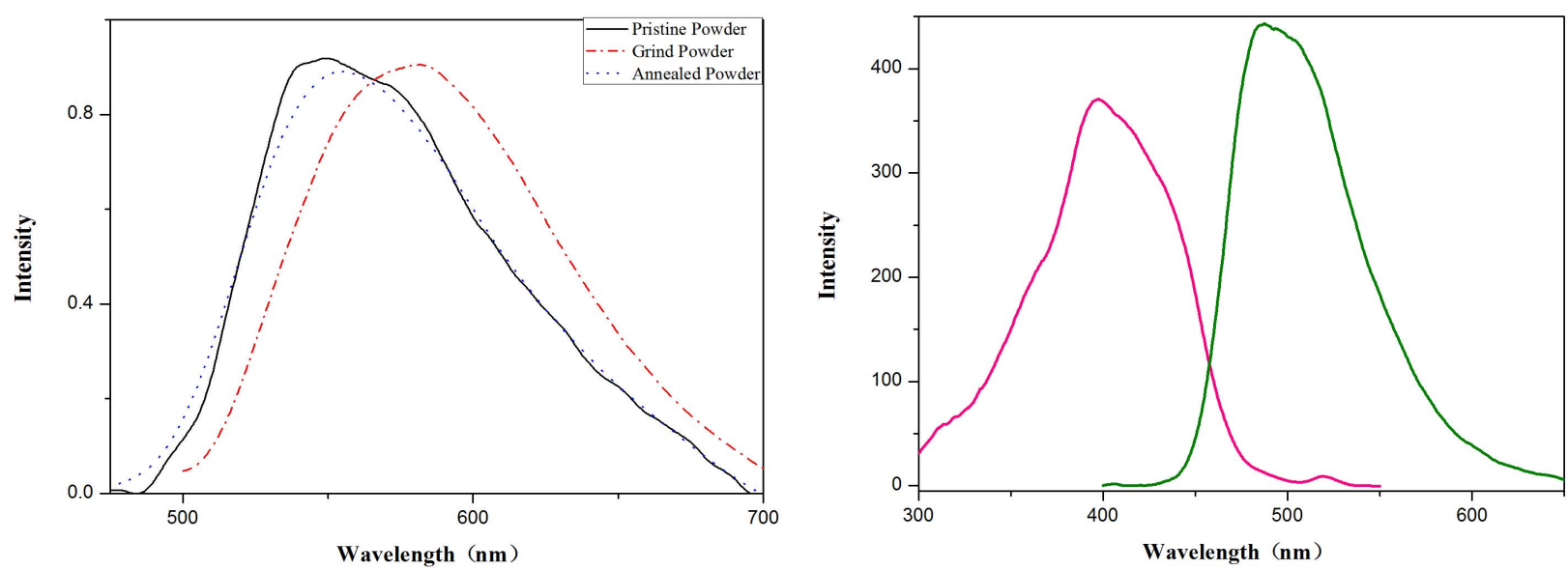

Figure 5. Normalized photoluminescence spectra for TOPV1: Left: solid line measured for original state, red line measured after grinding, blue dotted line measured for ground sample annealed at $180^{\circ} \mathrm{C}$ for $5 \mathrm{~min}$. Right: Photoluminescence spectra: absorption (purple), emission (green).
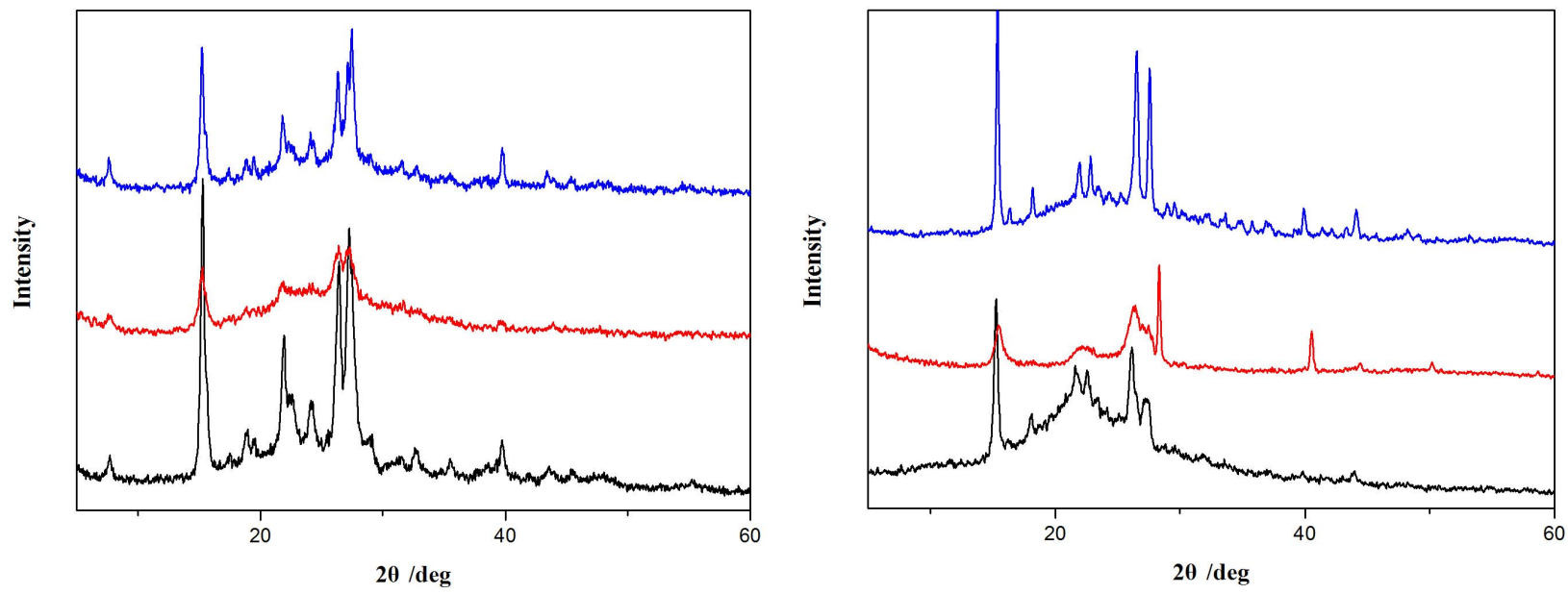

Figure 6. Power XRD patterns for TOPV1 (left) and TOPV2 (right). Black: original sample; Red: ground sample; Blue: annealed sample.

system could change its existing form from a solution or well dispersed state in the pure THF to aggregated particles in the mixtures with high water content. As shown in Figure 7, if the water fraction is less than $60 \%$, the PL intensity of the compound is very weak. The increase in PL intensity could be attributed to the AIE effect caused by the formation of molecular aggregates when adding water to the solution. Therefore, we conclude that TOPV 2 has both aggregation induced emission and piezofluorochromic characteristics and is a PAIE material.

A comparison of the photoluminescence properties of the two TOPV compounds reveals that the position of the cyano substituent has a significant effect. Although, the origin of the effect is unclear, the effect may derive from the molecular packing in the solid state. We also suggest that the peripheral thiomethyl substituents may amplify the piezoflurochromic properties of OPV materials.

\section{Conclusion}

We have synthesized the first examples of cyano oligo (p-phenylene vinylene) derivatives with thiomethyl groups at peripheral positions. We have also studied the impact of the length of $\pi$-bridge in OPV and the position of the -CN groups on their piezochromic properties. The new thiomethyl and cyano OPV compounds exhibit fluorescence emission propertie, and can reversibly switch upon pressing or annealing owing to crystalline amorphous phase transformation. These extraordinary properties make those two compounds promising candidates for stimuli-responsive, smart, luminescent materials for variety applications. Also, it is exciting to find the compound with $-\mathrm{CN}$ groups close to the central aromatic ring (TOPV2) is a PAIE material. The structure-property relationship established here would be helpful in determining 


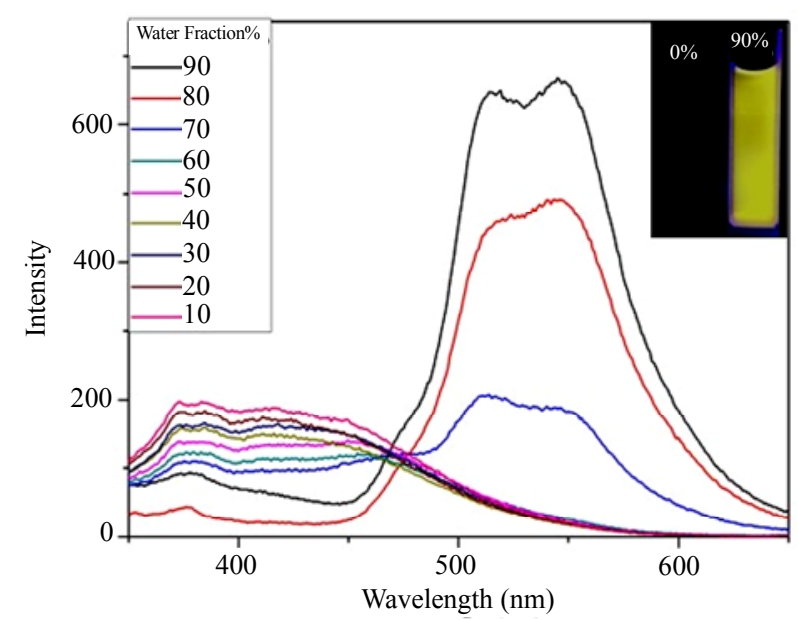

Figure 7. PL spectra for TOPV2 in water/THF mixtures. The insets depict emission images in pure THF and 90:10 water:THF solvent mixture under $365 \mathrm{~nm}$ UV light.

the mechanism for the PAIE process.

\section{Acknowledgements}

This research was supported by the Ministry of Education of China (Grant No. 20126203120002 of "Specialized Research Fund for the Doctoral Program of Higher Education, New Teachers" in the year 2012) and Northwest Normal University (Grant No. NWNU- LKQN-122 of "New Teacher Scientific Research Improvement Program").

\section{REFERENCES}

[1] D. A. Davis, A. Hamilton, J. Yang, J. S. Moore and N. R. Sottos, "Force-Induced Activation of Covalent Bonds in Mechanoresponsive Polymeric Materials," Nature, Vol. 459, No. 3, 2009, pp. 68-72. doi:10.1038/nature07970

[2] J. W. Chung, Y. You, H. S. Huh, B. K. An, S. J. Yoon, S. H. Kim, S. W. Lee and S. Park, "Shear- and UV-Induced Fluorescence Switching in Stilbenic $\pi$-Dimer Crystals Powered by Reversible [2 + 2] Cycloaddition," Journal of the American Chemical Society, Vol. 131, No. 23, 2009, pp. 8163-8172. doi:10.1021/ja900803d

[3] Y. Sagara, T. Mutai, I. Yoshikawa and K. Araki, "Material Design for Piezochromic Luminescence: HydrogenBond-Directed Assemblies of a Pyrene Derivative," Journal of the American Chemical Society, Vol. 129, No. 6, 2007, p. 1520. doi:10.1021/ja0677362

[4] Y. Muramatsu, T. Yamamoto, M. Hasegawa, T. Yagi and H. Koinuma, "Piezochromic Behaviour of Regioregular Poly(3-hexylthiophene-2,5-diyl) and Poly(5,8-dihexadecyloxyanthraquinone-1,4-diyl)," Polymer, Vol. 42, No. 15, 2001, pp. 6673-6675. doi:10.1016/S0032-3861(01)00136-7

[5] Y. Dong, J. W. Y. Lam, A. Qin, J. Liu, Z. Li, B. Z. Tang,
J. Sun and H. S. Kwok, "Aggregation-Induced Emissions of Tetraphenylethene Derivatives and Their Utilities as Chemical Vapor Sensors and in Organic Light-Emitting Diodes," Applied Physics Letters, Vol. 91, No.1, 2007, Article ID: 011111. doi:10.1063/1.2753723

[6] Z. Ning, Z. Chen, Q. Zhang, Y. Yan, S. Qian, Y. Cao and H. Tian, "Aggregation-Induced Emission (AIE)-Active Starburst Triarylamine Fluorophores as Potential NonDoped Red Emitters for Organic Light-Emitting Diodes and $\mathrm{Cl}_{2}$ Gas Chemodosimeter," Advanced Functional Materials, Vol. 17, No. 18, 2007, pp. 3799-3807. doi:10.1002/adfm.200700649

[7] B. R. Crenshaw, M. Burnworth, D. Khariwala, A. Hiltner, P. T. Mather, R. Simha and C. Weder, "Deformation-Induced Color Changes in Mechanochromic Polyethylene Blends," Macromolecules, Vol. 40, No. 7, 2007, pp. 2400 2048. doi:10.1021/ma062936j

[8] M. Kinami, B. R. Crenshaw and C. Weder, "Polyesters with Built-In Threshold Temperature and Deformation Sensors," Chemistry of Materials, Vol. 18, No. 4, 2006, pp. 946-955. doi:10.1021/cm052186c

[9] S. J. Toal, K. A. Jones, D. Magde and W. C. Trogler, "Luminescent Silole Nanoparticles as Chemoselective Sensors for Cr(VI)," Journal of the American Chemical Society, Vol. 127, No. 33, 2005, pp. 11661-11665. doi:10.1021/ja052582w

[10] S. Hirata and T. Watanabe, "Reversible Thermoresponsive Recording of Fluorescent Images (TRF)," Advanced Materials, Vol. 18, No. 20, 2006, pp. 2725-2729. doi:10.1002/adma.200600209

[11] S. J. Lim, B. K. An, S. D. Jung, M. A. Chung and S. Y. Park, "Photoswitchable Organic Nanoparticles and a Polymer Film Employing Multifunctional Molecules with Enhanced Fluorescence Emission and Bistable Photochromism," Angewandte Chemie International Edition, Vol. 43, No. 46, 2004, pp. 6346-6350. doi:10.1002/anie.200461172

[12] M. Irie, T. Fukaminato, T. Sasaki, N. Tamai and T. Kawai, "Organic Chemistry: A Digital Fluorescent Molecular Photoswitch," Nature, Vol. 420, No. 6917, 2002, pp. 759760. doi:10.1038/420759a

[13] G. S. Papaefstathiou, Z. Zhong, L. Geng and L. R. MacGillivray, "Coordination-Driven Self-Assembly Directs a Single-Crystal-to-Single-Crystal Transformation That Exhibits Photocontrolled Fluorescence," Journal of the American Chemical Society, Vol. 126, No. 30, 2004, pp. 9158-9159. doi:10.1021/ja047819n

[14] A. Kishimura, T. Yamashita, K. Yamaguchi and T. Aida, "Rewritable Phosphorescent Paper by the Control of Competing Kinetic and Thermodynamic Self-Assembling Events," Nature Materials, Vol. 4, No. 1, 2005, pp. 546549. doi:10.1038/nmat1401

[15] Z. Chi, X. Zhang, B. Xu, X. Zhou, C. Ma, Y. Zhang, S. Liu and $\mathrm{J} . \mathrm{Xu}$, "Recent Advances in Organic Mechanofluorochromic Materials," Chemical Society Reviews, Vol. 41, No. 10, 2012, pp. 3878-3896. doi: $10.1039 / \mathrm{c} 2 \operatorname{cs} 35016 \mathrm{e}$

[16] S. Mizukami, H. Hojou, K. Sugaya, E. Koyama, H. 
Tokuhisa, T. Sasaki and M. Kanesato, "Fluorescence Color Modulation by Intramolecular and Intermolecular $\pi-\pi$ Interactions in a Helical Zinc(II) Complex," Chemistry of Materials, Vol. 17, No. 1, 2005, pp. 50-56.

doi:10.1021/cm049744s

[17] C. Lowe and C. Weder, "Oligo( $p$-phenylene vinylene) Excimers as Molecular Probes: Deformation-Induced Color Changes in Photoluminescent Polymer Blends," $A d$ vanced Materials, Vol. 14, No. 22, 2002, pp. 1625-1629. doi:10.1002/1521-4095(20021118)14:22<1625::AID-AD MA1625>3.0.CO;2-Q

[18] J. Kunzelman, M. Kinami, B. R. Crenshaw, J. D. Protasiewicz and C. Weder, "Oligo(p-phenylene vinylene)s as a 'New' Class of Piezochromic Fluorophores," Advanced Materials, Vol. 20, No. 1, 2008, pp. 119-122. doi:10.1002/adma.200701772
[19] C. Löwe and C. Weder, "Synthesis and Properties of Photoluminescent 1,4-Bis-( $\alpha$-cyano-4-methoxystyryl)benzenes,' Synthesis, Vol. 9, No. 9, 2002, pp. 1185-1190. doi: $10.1055 / \mathrm{s}-2002-32537$

[20] M. A. Garcia-Garibay, "Molecular Crystals on the Move: From Single-Crystal-to-Single-Crystal Photoreactions to Molecular Machinery," Angewandte Chemie International Edition, Vol. 46, No. 47, 2007, pp. 8945-8947. doi:10.1002/anie. 200702443

[21] B.-K. An, S. K. Kwon, S. D. Jung and S. Y. Park, "Enhanced Emission and Its Switching in Fluorescent Organic Nanoparticles," Journal of the American Chemical Society, Vol. 124, No. 48, 2002, pp. 14410-14415. doi:10.1021/ja0269082 